\title{
5th Workshop on innovative mouse models, IMM 2009, June 25-26, Leiden, The Netherlands: a meeting report
}

\author{
Marian van Roon
}

Received: 17 July 2009/Accepted: 20 July 2009/Published online: 19 August 2009

(C) The Author(s) 2009. This article is published with open access at Springerlink.com

On June 25-26, 2009 the 5th Workshop on innovative mouse models was held in the Leiden University Medical Center, Leiden, The Netherlands. The primary goal of this two-day workshop has always been to bring together a diverse group of scientists interested in advanced genome alteration approaches in the mouse, including key developers of emerging technologies as well as researchers who wish to apply and assess these new approaches. The workshop encourages an in-depth and unvarnished discussion of these technologies and novel developments in a very open and informal way, easy accessible for young and senior researchers. The organising committee consisting of Jos Jonkers (NKI, Amsterdam, The Netherlands), Paul Krimpenfort (NKI, Amsterdam, The Netherlands), Werner Müller (University of Manchester, UK), Els Robanus Maandag (LUMC, Leiden, The Netherlands), Hein te Riele (NKI, Amsterdam, The Netherlands), Marian van Roon (AMC, Amsterdam, The Netherlands) and Sjef Verbeek (LUMC, Leiden, The Netherlands) all, except for Werner Müller, participated in the group of Anton Berns, NKI, Amsterdam and got acquainted with transgenic technologies and mouse models in his lab. From 2003 onwards they decided to bring together all

M. van Roon $(\square)$

Animal Research Institute AMC, S0-136, Academic

Medical Center, Meibergdreef 67, 1105 BK Amsterdam,

The Netherlands

e-mail:m.a.vanRoon@amc.uva.nl researchers in these fields in the characteristic informal, but outstanding workshops on IMM. The workshops grew from a European meeting to an international workshop, bringing together 150-200 scientists (see http://workshop.nki.nl). This year, during the first lustrum, topics included: induced Pluripotent Stemcells, Sleeping Beauty transposon mutagenesis, large scale generation of conditional knockouts, in vivo RNAi and mosaic mouse models. The topics were covered during the first day of the workshop by excellent and outstanding keynote speakers, while the second day the same topics were discussed by young scientists in high quality, short presentations.

All participants were welcomed in the beautiful and well equipped Lecture Hall of the Leiden University Medical Center by the initiator of the IMM workshops Sjef Verbeek. Sjef had a warm welcome for all of the participants and expressed his gratitude to all the sponsors of this workshop: European Science Foundation (ESF), the International Society for Transgenic Technologies (ISTT), COST, NWO-ZonMw, LUMC, NKI-AvL and AMC, who made the workshop possible against the low registration fee.

The first session, 28 years of genetically engineered mice: quo vadis?, was kicked off by Anton Berns from The Netherlands Cancer Institute. Anton was the pioneer of genetic modification of mice in The Netherlands and almost all of the organising committee learned about genetic modification and 
mouse models in his lab. Anton gave a nice review of the milestones of transgenic technology in simple and funny cartoons. He showed the evolution of mouse models by unnatural selection and focussed on the points that need work:- the way the system works and the access we should have to transgenic and $\mathrm{KO}$ strains - the lack of phenotyping - the genetic background of the mice generated (FVB and C57Bl/6) the generation of compound mutants which is currently quite slow and should force us to make compound ES cells and derive mice directly and to make more use of somatic gene transfer-implementation of versatile reporter systems. Berns urged us to join forces and generate support facilities, sufficient funding, repositories for ES cells and sperm, standardization and high throughput sites and finally all of us should try to increase acceptance of genetically modified organisms. During the discussion Berns got support for his consortia initiatives but they should be more interactive with the users and be more diverse, using more technologies.

The second presentation was by Francis Stewart (TU Dresden) who explained to us the technique of recombineering: DNA engineering using recombination in E. coli, fast, easy, and suitable for complex alleles and site specific recombination. Francis explained in detail the process and the use of 'other' coli strains (pSC101), pir+ strains to lower the background, and the importance of testing antibiotics concentrations. He showed the easiness of recombineering, which was later on in the workshop confirmed by several enthusiastic users, and promised us that the "future is ligation-free". Francis also discussed some modifications of already existing techniques like the development of site specific recombination system Dre/rox (P1) (Sauer and McDermott 2004). The Dre/ rox is a good partner in recombination with Cre/lox, the two do not show cross-talk and thus can be used together. Another modification is the Flpo; a codon optimized form of Flpe, (Raymond and Soriano 2007). Stewart is developing a Flpo deleter mouse, which will be available soon. As an example of the High Throughput recombineering pipeline Stewart mentioned the making of BAC transgenes and targeting constructs in 96-well plates by 96-well plate electroporation, a process that will only take 3-4 days. Finally Stewart referred to the website of his company for detailed information on all these techniques and the used materials.
The third lecture from this first session was by Barry Rosen (Wellcome Trust Sanger Institute, Hinxton) who showed us the EUCOMM and KOMP pipelines of conditional KO's and deletion KO's and the process of design, construction (Gateway system) and targeting in C57B1/6N cells (originally from JM8 cells). In the pipeline they learned that promoterless constructs work very efficiently in targeting, that promoterdriven cassettes are fine as well, but that the promoter should be removed after homologous recombination and that the PGK-DTA negative selection greatly improves the promoter gene targeting efficiency from 7 to $42 \%$. During the discussion Barry had a hard time explaining why it takes so long for the community to receive targeted ES cells after applying for them. He promises a more efficient handling and urges people to keep on applying.

After lunch we did go on with session 2, the first out of three sessions entitled 'New technologies'. First was Austin Smith (Wellcome Trust Centre for Stem Cell Research, Cambridge) who talked about capturing pluripotency. Austin showed that for mouse ES cells LIF and BMP are dispensable and that only two factors, called $2 \mathrm{i}$, will keep the pluripotency of ES cells. Everything else is dispensable. He showed the response of the embryo to $2 \mathrm{i}$ by developing the ES cells to blastocysts and look at the formation of the epiblast and hypoblast. It turns out that $2 \mathrm{i}$ suppresses the emergence of the hypoblast and promotes the formation of the epiblast. $2 \mathrm{i}$ grown epiblasts make high grade chimeras and it was suggested that the epiblast may have no dependence on hypoblast. $2 \mathrm{i} /$ LIF is able to raise ES cells from all mouse strains, as an example NOD ES cells were shown. Rat embryonic stem cells can also be raised and grown on $2 \mathrm{i} /$ LIF/feeders and give rise to germline offspring, but they are trickier to grow than the mouse cells. Human "ES" cells cannot be maintained in $2 \mathrm{i}$ and are dependent on erk signalling. The question therefore remains if the human "ES" cells are true ES cells.

Smith was followed up by Andras Nagy (Mount Sinai Hospital, Toronto) who combined to new techniques: the formation of induced pluripotent stem cells (iPS) and transposon/transposase technology. Andras showed that fibroblasts can be reprogrammed by the combination of c-myc, klf4, oct 4 and sox2 (MKOS) to become iPS cells, which can than be used as therapeutic cells and disease models. The high risk of these cells is the remaining presence of 
the introduced transgenes and their possible effects on all kind of biological processes. Andras showed the use of transposon based transfection by using the PiggyBac transposon system. The system is very efficient and cotransduces the transposase and the GOI in the genome. The second time the transposase gets active, it removes the GOI but also alters it in such a way that reintegration is diminished by $50 \%$ (Wang et al. 2008).

Lars Zender (Helmholtz Centre for Infection Research, Braunschweig) talked about the integrative oncogenomic approaches for accelerated cancer gene discovery in hepatocellular carcinoma. He uses FAH-/- mice as a model system for liver damage and liver failure and combines it with the transposon/ transposase technology.

And last but not least for this first day Adam Dupuy (University of Iowa, USA) showed the use of the SleepingBeauty transposon system in oncogene activation and tumour suppressor gene inactivation in models of T-ALL. The big disadvantage of all of the transposon based systems of course remains the local hopping.

The day was closed with a well visited poster presentation, and enough time for informal discussions and get-to-getters with the keynote speakers. A nice walk through the sunny centre of Leiden, over the canal bridges and along the mills brought us to the Kamerlingh Omnes building were we had dinner in good company and a relaxed but inspiring atmosphere.

The second day of the workshop was devoted to short oral presentations selected from the poster abstracts. All of these can be found back in the workshop abstract book. The main focus was on the transposon systems, the use of rat spermatogonial stem cells (very nice contribution by Ivics), the efficient system of targeting to the Hprt locus and the lentiviral-mediated RNAi silencing. Frustrating was the report that the lentiviral vectors also undergo gene silencing after generation 6 , just when you managed to segregate the multiple sites of integration! Personally I was very much impressed by the high quality of the short presentations and the excellent time management of the speakers. It made it a very nice second day. The workshop was closed with a forum discussion, which really made it to a "WORK"shop. Moderator Hein te Riele showed in an elegant way that there was no forum, but that all the participants formed the forum and that the technique of the Leiden lecture hall enabled us to follow the discussion on a big screen. It was a stimulating discussion on very diverse topics. We discussed the use and efficiency of the big consortia and urged them to be more interactive with their customers and to really set up a good system for sharing their outcomes with the scientific community. We compared the use of ES cells and iPS cells and discussed the generation of compound mice and/or compound ES cells. Should everybody be willing to do all transgenic technology by themselves or should we outsource techniques to the experts? How can we really share animal models once they are published and how do we get new techniques and materials in our laboratories? And finally, what are the risks of working with genetically modified models, how should we make genetic modification acceptable to the public and why did we announce the workshop as Innovative Models in the LUMC and left out Mouse? These informal discussions in an open and respectful atmosphere kept participants bound to their seats till the closing and formed the cherries on the workshop cake and made it a pleasure to organise. All participants, sponsors, and speakers are thanked for their excellent contributions. We hope to see you all again on the 6th IMM workshop.

Open Access This article is distributed under the terms of the Creative Commons Attribution Noncommercial License which permits any noncommercial use, distribution, and reproduction in any medium, provided the original author(s) and source are credited.

\section{References}

Raymond CS, Soriano P (2007) High-efficiency FLP and PhiC31 site-specific recombination in mammalian cells. PLoS ONE 2(1):e162

Sauer B, McDermott J (2004) DNA recombination with a heterospecific Cre homolog identified from comparison of the pac-c1 regions of P1-related phages. Nucleic Acids Res 32(20):6086-6095 Print 2004

Wang W, Lin C, Lu D, Ning Z, Cox T, Melvin D, Wang X, Bradley A, Liu P (2008) Chromosomal transposition of PiggyBac in mouse embryonic stem cells. Proc Natl Acad Sci 105(27):9290-9295 Epub 2008 Jun 25 